\title{
Kinetic-energy functionals studied by surface calculations
}

\author{
Vitos, Levente; Skriver, Hans Lomholt; Kollár, J.
}

\section{Published in:}

Physical Review B

Link to article, DOI:

10.1103/PhysRevB.57.12611

Publication date:

1998

Document Version

Publisher's PDF, also known as Version of record

Link back to DTU Orbit

Citation (APA):

Vitos, L., Skriver, H. L., \& Kollár, J. (1998). Kinetic-energy functionals studied by surface calculations. Physical Review B, 57(19), 12611-12615. https://doi.org/10.1103/PhysRevB.57.12611

\section{General rights}

Copyright and moral rights for the publications made accessible in the public portal are retained by the authors and/or other copyright owners and it is a condition of accessing publications that users recognise and abide by the legal requirements associated with these rights.

- Users may download and print one copy of any publication from the public portal for the purpose of private study or research.

- You may not further distribute the material or use it for any profit-making activity or commercial gain

- You may freely distribute the URL identifying the publication in the public portal

If you believe that this document breaches copyright please contact us providing details, and we will remove access to the work immediately and investigate your claim. 


\title{
Kinetic-energy functionals studied by surface calculations
}

\author{
L. Vitos and H. L. Skriver \\ Center for Atomic-scale Materials Physics and Department of Physics, Technical University of Denmark, DK-2800 Lyngby, Denmark \\ J. Kollár \\ Research Institute for Solid State Physics, P.O. Box 49, H-1525 Budapest, Hungary
}

(Received 10 November 1997)

\begin{abstract}
The self-consistent jellium model of metal surfaces is used to study the accuracy of a number of semilocal kinetic-energy functionals for independent particles. It is shown that the poor accuracy exhibited by the gradient expansion approximation and most of the semiempirical functionals in the low density, high gradient limit may be subtantially improved by including locally a von Weizsäcker term. Based on this, we propose a simple one-parameter Padé's approximation, which reproduces the exact Kohn-Sham surface kinetic energy over the entire range of metallic densities. [S0163-1829(98)03719-9]
\end{abstract}

\section{INTRODUCTION}

Within density functional theory, ${ }^{1}$ the kinetic energy of the noninteracting particles, defined as the sum of oneelectron kinetic energies, may be determined exactly from the one-electron wave functions $\psi_{j}(\mathbf{r})$ as

$$
T^{\mathrm{KS}}[n]=\sum_{j}^{\mathrm{occ}} \int d \mathbf{r} \psi_{j}^{*}(\mathbf{r})\left(-\frac{\hbar^{2}}{2 m} \nabla^{2}\right) \psi_{j}(\mathbf{r}),
$$

which is an implicit functional of the electron density given by

$$
n(\mathbf{r})=\sum_{j}^{\text {occ }}\left|\psi_{j}(\mathbf{r})\right|^{2}
$$

The application of the orbital-based expression (1) in total energy calculations requires a knowledge of the complete self-consistent solution to the Kohn-Sham equations. ${ }^{2}$ Unfortunately, the exact self-consistent solution of the Kohn-Sham problem is a time-consuming task, and in many cases, such as $a b$ initio molecular dynamics calculations, ${ }^{3-5}$ it becomes the time limiting step. Furthermore, there are total energy methods that derive the charge density by an alternative route, ${ }^{6,7}$ and in these cases the calculation of one-electron orbitals to determine the kinetic energy would form an inefficient backward step. If, however, there existed an accurate, explicit density-dependent kinetic energy functional, both the computational effort of the self-consistent procedures and of calculating the kintic energy and, hence, the total energy, could be considerably reduced.

During the past decade many attempts have been made to develop accurate orbital-free semilocal $^{8-12}$ and nonlocal $^{4,13-15}$ kinetic energy functionals. Although most of the nonlocal functionals may reproduce with high accuracy the exact Kohn-Sham results, including the quantum mechanical shell structure of the atomic densities, ${ }^{15}$ their application to realistic three-dimensional extended systems raises numerical difficulties, and in the present work we therefore focus on the semilocal kinetic energy functionals. Further, an orbital-free kinetic energy functional should give accurate density profiles as well as kinetic energies. However, the accuracy of the semilocal density functionals in the calculation of density profiles is usually considerably lower than that obtained by insertion of exact densities. ${ }^{16}$ We therefore consider only the latter case, i.e., we use the exact selfconsistent densities obtained using the Kohn-Sham scheme ${ }^{2}$ within the local density approximation for the exchangecorrelation energy in order to test the accuracy of the semilocal kinetic energy functionals.

Most of the above mentioned semilocal and nonlocal functionals, with a few exceptions, ${ }^{15}$ have been designed and tested for use in atomic calculations and, as it turns out, they are not well suited for surface calculations. The principal aim of the present study is to establish the accuracy of a number of first-principles and representative semiempirical kinetic energy functionals and to isolate those factors that are crucial for metal surfaces but irrelevant for atoms and molecules. To have an accurate reference system, we consider the selfconsistent jellium surface model by Lang and Kohn, ${ }^{17}$ within which the 3D Kohn-Sham problem reduces to a onedimensional problem where the numerical approximations may be kept at a minimum. Such a semi-infinite jellium surface is characterized by the electron density parameter $r_{s}$ $=\left(3 / 4 \pi n_{b}\right)^{1 / 3}$ given in terms of the bulk density $n_{b}$. The present study covers the physically interesting density range $r_{s}=2-6$ and uses Wigner's exchange-correlation functional ${ }^{18}$ as in the original jellium surface calculations. ${ }^{17}$

\section{THE DENSITY GRADIENT EXPANSION}

For slowly varying densities the noninteracting kinetic energy functional $T[n]$ may be given in the form of a density gradient expansion (GE) ${ }^{16}$ In terms of the kinetic energy density, defined by

$$
T[n] \equiv \int t[n] d \mathbf{r},
$$

this expansion can be written in the following form:

$$
t_{\mathrm{GE}}[n]=t^{(0)}(n)+t^{(2)}[n]+t^{(4)}[n]+\cdots,
$$


TABLE I. The errors of various kinetic energy functionals for self-consistent jellium surface profiles corresponding to $r_{s}=2-6$ in percent of the exact Kohn-Sham surface kinetic energy. $T_{\mathrm{GE}}^{0}, T_{\mathrm{GE}}^{2}$, and $T_{\mathrm{GE}}^{4}$ are the zeroth-, second-, and fourth-order gradient expansion functionals (Ref. 16), $T_{\mathrm{TF}(1 / 4) W}$ is the ThomasFermi-von Weizsäcker functional for $\lambda=1 / 4$ (Ref. 20), $T_{L}$ the locally truncated functional by Pearson and Gordon (Ref. 12), $T_{\mathrm{LC}}$ the generalized gradient approximation functional by Perdew et al. (Ref. 27), and parametrized by Lembarki and Chermette (Ref. 24), $T_{\mathrm{DK}}$ the Padé form by DePristo and Kress (Ref. 8), $T_{\mathrm{GE} / W}$ the functional from Eq. (11), and $T_{3,2}$ the Padé form given by Eq. (13).

\begin{tabular}{cccccccccc}
\hline \hline$r_{s}$ & $T_{\mathrm{GE}}^{0}$ & $T_{\mathrm{GE}}^{2}$ & $T_{\mathrm{GE}}^{4}$ & $T_{\mathrm{TF}(1 / 4) W}$ & $T_{L}$ & $T_{\mathrm{LC}}$ & $T_{\mathrm{DK}}$ & $T_{\mathrm{GE} / W}$ & $T_{3,2}$ \\
\hline 2.0 & -11 & -2 & -1 & 8 & -2 & -2 & -3 & 0.0 & -0.2 \\
2.5 & -17 & -5 & -2 & 11 & -4 & -4 & -5 & 0.5 & -0.1 \\
3.0 & -24 & -8 & -4 & 13 & -7 & -8 & -8 & 0.6 & 0.1 \\
3.5 & -35 & -13 & -6 & 15 & -12 & -13 & -13 & 2.9 & 0.3 \\
4.0 & -51 & -21 & -10 & 18 & -21 & -22 & -21 & 5.5 & 0.4 \\
4.5 & -77 & -33 & -17 & 21 & -33 & -36 & -32 & 8.7 & 0.5 \\
5.0 & -121 & -56 & -29 & 26 & -57 & -60 & -53 & 12.3 & 0.4 \\
5.5 & -223 & -108 & -55 & 37 & -109 & -117 & -101 & 17.1 & 0.3 \\
6.0 & -650 & -329 & -170 & 74 & -333 & -359 & -302 & 29.6 & -0.6 \\
\hline \hline
\end{tabular}

where the zeroth order term $t^{(0)}(n)$ is the Thomas-Fermi energy density which depends only on the density $n(\mathbf{r})$ at the point $\mathbf{r}$. All other terms involve the gradient $\nabla n$ and higher order derivatives of the charge density, hence they include some nonlocal contributions from the electron density in the immediate vicinity of $\mathbf{r}$.

The errors of the expansion (4) including one $\left(T_{\mathrm{GE}}^{0}\right)$, two $\left(T_{\mathrm{GE}}^{2}\right)$, or three $\left(T_{\mathrm{GE}}^{4}\right)$ terms relative to the exact Kohn-Sham value for jellium surfaces are shown in Table I and in Fig. 1. Here and in the following we use the definition (3) for each component of the expansion as well as for the total kinetic energy functionals. It is seen that for the high and intermediate densities, i.e., $r_{s}=2-4.5$, the error of the ThomasFermi functional $T_{\mathrm{GE}}^{0}$ is to a large degree corrected by the second- $\left(T^{(2)}\right)$ and fourth-order $\left(T^{(4)}\right)$ terms, showing the rapid convergence of the expansion. Note that this is not convergence in the strict sense because in the gradient expansion for surface density profiles the sixth- and higherorder terms diverge if the exponentially decreasing densities are inserted. ${ }^{16}$ On the other hand, in the low density limit the

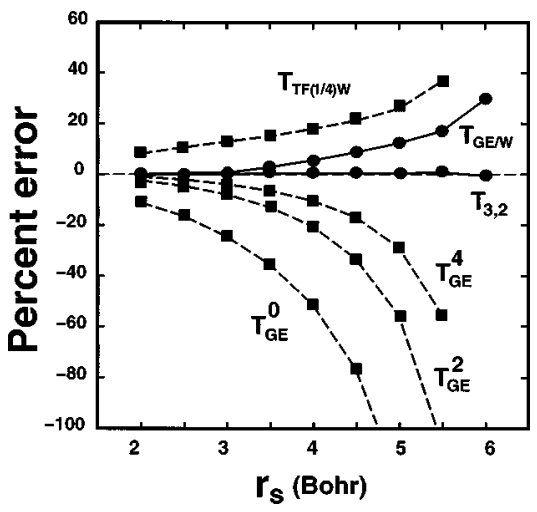

FIG. 1. Errors of the surface kinetic energy obtained by different kinetic energy functionals relative to the exact Kohn-Sham kinetic energy versus $r_{s} . T_{\mathrm{GE}}^{0}, T_{\mathrm{GE}}^{2}$, and $T_{\mathrm{GE}}^{4}$ are the zeroth-, second-, and fourth-order gradient expansion functionals (Ref. 16), $T_{\mathrm{TF}(1 / 4) W}$ is the Thomas-Fermi-von Weizsäcker functional (Ref. 20), $T_{\mathrm{GE} / W}$ the functional from Eq. (11), and $T_{3,2}$ the Padé form from Eq. (13). error of the fourth-order gradient expansion $T_{\mathrm{GE}}^{4}=T^{(0)}$ $+T^{(2)}+T^{(4)}$ is still very large $(170 \%)$. At this stage we mention that the accuracy of the fourth-order gradient expansion for the Lang-Kohn density profiles, especially for $r_{s}$ $>3$, is considerably better than that found for other less realistic surface models, such as the linear potential approximation. ${ }^{16,19}$

To understand the failure of the gradient expansion for surfaces at high $r_{s}$ values, we now examine its local convergence in terms of the semilocal variables,

$$
s[n] \equiv \frac{|\nabla n|}{2 k_{F} n} \quad \text { and } q[n] \equiv \frac{\left|\nabla^{2} n\right|}{2 k_{F}|\nabla n|},
$$

where $k_{F}=\left(3 \pi^{2} n\right)^{1 / 3}$ is the Fermi wavelength. As stated in the original work by Hohenberg and Kohn, ${ }^{1}$ the criterion for convergence of the expansion (4) is that both of these dimensionless quantities are well below unity. This condition is generally satisfied in bulk metals and in the inner part of isolated atoms. ${ }^{12}$ However, for the exponentially decaying densities, which occur in the exterior region of atoms ${ }^{12}$ and at metal surfaces, the condition is violated, and in fact for these systems both $s$ and $q$ go to infinity.

In Fig. 2 we plot the scaled gradient $s[n]$ versus the distance $z$ from the edge of the positive jellium background (in units of $k_{F}$ ) for two self-consistent density profiles. It is observed that $s[n]$ increases from zero to infinity when the surface is reached. Moreover, as the bulk density decreases, i.e., $r_{s}$ increases, the scaled gradient $s[n]$ viewed as a function of $z$ rises rapidly while the density is still finite. From the inset, which shows the relative kinetic energy density $t_{\mathrm{GE}}^{2}[n] / t_{\mathrm{GE}}^{2}\left[n_{b}\right]$ calculated using the second order gradient expansion and plotted as a function of the scaled gradient $s[n]$, it is seen that, within this approximation, the kinetic energy density for $r_{s}=2$ decreases more rapidly with the scaled gradient than for $r_{s}=6$. Hence, in the large $r_{s}$ regime a sizeable contribution to the surface kinetic energy comes from spatial regions where the scaled gradient is large, and therefore the error of the gradient expansion increases with $r_{s}$. 


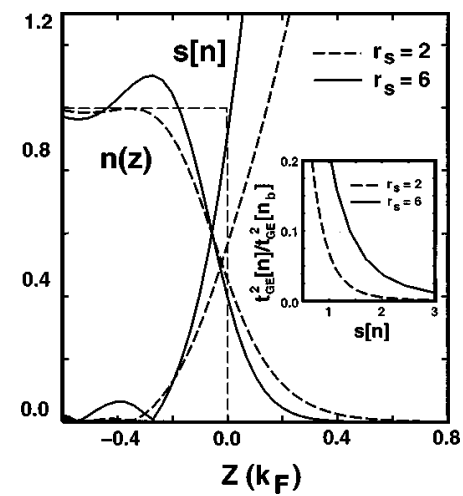

FIG. 2. The self-consistent density profiles $n(z)$ (in units of the bulk density $n_{b}$ ) and the corresponding scaled gradient $s[n]$ for $r_{s}=2$ and $r_{s}=6$. The inset shows the relative kinetic energy density $t_{\mathrm{GE}}^{2}[n] / t_{\mathrm{GE}}^{2}\left[n_{b}\right]$ calculated within the second order gradient approximation versus the scaled gradient $s[n]$ for the same $r_{s}$ values.

It is clear that the shortcoming of the gradient expansion in surface calculations is caused by the combination of a large gradient and a finite density, which occurs near a surface. Such a combination is particular to surfaces and does not play a role at the outer regions of isolated atoms where the density decreases much more rapidly. This, on the other hand, means that the fitting procedures used to design kinetic energy functionals for use in atomic calculations never test combinations of large gradients and finite densities. It is therefore no surprise that these semiempirical functionals do not work well in surface calculations.

\section{KINETIC ENERGY FUNCTIONALS}

To put the analysis of the preceding section on a quantitative basis, we test in the following a number of kinetic energy functionals in calculations of surface kinetic energies and discuss their accuracy. Based on this, we propose a particularly simple functional that for surfaces yields essentially exact, $<1 \%$, results, and that for atoms is of the same accuracy as the second order gradient expansion.

\section{A. The Thomas-Fermi-von Weizsäcker functional}

In the Thomas-Fermi-von Weizsäcker model, ${ }^{20}$ which may be considered a modified second order gradient expansion, the strength of the second order gradient term $t^{(2)}[n]$ is controlled by an adjustable parameter $\lambda$, i.e.,

$$
t_{\mathrm{TF}(\lambda) W}[n]=t^{(0)}(n)+9 \lambda t^{(2)}[n] .
$$

For $\lambda=1 / 9$, this functional is equivalent to the second order gradient expansion. Chizmeshya and Zaremba ${ }^{21}$ tested the Thomas-Fermi-von Weizsäcker functional in jellium surface calculations and found that $\lambda=1 / 4$ represented an optimal value in the density range $r_{s}=2-6$. In Table I and Fig. 1 we show jellium surface results obtained by Eq. (6) for $\lambda=1 / 4$. It is seen that the $T_{\mathrm{TF}(1 / 4) W}[n]$ functional leads to substantially improved results relative to the gradient expansion in the high $r_{s}$ range but to considerably higher errors in the low $r_{s}$ range. We found by modifying $\lambda$ that the average error in the surface kinetic energy could not be reduced below approximately $10 \%$.

\section{B. The locally truncated GE functional}

Pearson and Gordon ${ }^{12}$ solved the divergence problem by a local truncation of the gradient expansion. That is, at each point in space the number of terms included in the series is determined by a local criterion based on the properties of an asymptotic series. In practice, this means that if the fourth order term is larger than the second order term, the fourth order term is discarded, otherwise it is kept. As a result, the locally truncated functional $t_{L}[n]$ is identical to Eq. (4) for slowly varying densities and reduces to the Thomas-Fermi term in the rapidly varying limit.

The $T_{L}[n]$ functional has been studied for a large number of atoms and molecules, ${ }^{12,22}$ and in all cases it gives better results for the total kinetic energy than a globally truncated gradient expansion. Its success may be assigned to the fact that for atoms and molecules the error caused by the Thomas-Fermi term in the large gradient limit, i.e., in the asymptotic region, is lower than that caused by an expansion that also includes the semilocal terms. However, in the case of a metal surface, an important contribution to the surface kinetic energy comes from regions of high gradients where the density is still finite, and such systems are not expected to be described properly by a locally truncated functional.

In Table I, the sixth column shows the results for the surface kinetic energy obtained by means of the $T_{L}[n]$ functional. The errors of this functional are only slightly higher than those obtained by the second order gradient expansion, and we may therefore conclude that the reduction of errors caused by including the fourth order term in the gradient expansion (4) comes mainly from regions where the gradient expansion is not valid.

\section{Functionals based on a gradient enhancement factor}

A widely used family of semiempirical kinetic energy functionals based on a conjointness of the kinetic and exchange energy has been introduced by Lee et al. ${ }^{23}$ These functionals may be written in the form

$$
t_{\mathrm{LLP}}[n]=t^{(0)}(n) F_{\mathrm{LLP}}(s),
$$

where $F_{\text {LLP }}(s)$ is an explicitly gradient dependent enhancement factor chosen to be the same as the one used in the corresponding exchange energy functional. ${ }^{23,24}$ The coefficients of this enhancement factor may be determined from exact atomic kinetic energies. This family of functionals has been tested for atoms ${ }^{25}$ and molecules, ${ }^{26}$ and it leads to better agreement with the exact values than the gradient expansion.

In the present test calculations with the $T_{\mathrm{LLP}}[n]$ functional, we use an enhancement factor $F_{\mathrm{LC}}$ of the functional form given by Perdew et $a .^{27}$ in their generalized gradient approximation to the exhange energy but fitted to atomic kinetic energies by Lembarki and Chermette. ${ }^{24}$ In Fig. 3 we plot a number of gradient factors including $F_{\mathrm{LC}}$ as functions of

$$
x[n] \equiv t^{(2)}[n] / t^{(0)}(n)=(5 / 27) s^{2}[n] .
$$

We note that for the second order gradient expansion, which is identical to the generalized gradient approximation for the kinetic energy, ${ }^{28}$ written in the form (7), we have $F_{\mathrm{GE}}^{2}(s)$ $=1+(5 / 27) s^{2}$. From Fig. 3 it is seen that for low $x$ values 


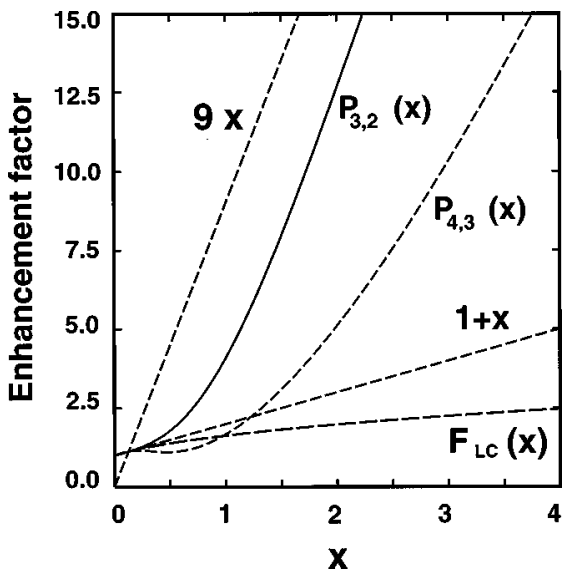

FIG. 3. Comparison of gradient enhancement factors of the various kinetic energy functionals. The dashed lines are the von Weizsäcker $(9 x)$, the DePristo and Kress $\left(P_{4,3}(x)\right)$, the second order gradient expansion $(1+x)$, and the Lembarki and Chermette $\left(F_{\mathrm{LC}}(x)\right)$ functionals. The solid line corresponds to the Pade form $\left(P_{3,2}\right)$ from Eq. (13).

$F_{\mathrm{LC}}(x)$ behaves like the second order gradient expansion, while for high $x$ values it falls below $F_{\mathrm{GE}}^{2}(x)$ and in the $x \rightarrow \infty$ limit it tends to $x^{-1} .^{27}$

The results obtained for the surface kinetic energy using the Lembarki-Chermette functional $T_{\mathrm{LC}}[n]$ are listed in the seventh column of Table I. The values are very close to those found using the second order gradient expansion, which shows that the high gradient limit is not properly included in this functional. We emphasize that the family of semiempirical kinetic energy functionals based on the conjointness argument of Lee et al. ${ }^{23}$ is unable to reproduce the high gradient limit $s \rightarrow \infty$, since the gradient enhancement factor $F(s)$ for the exchange functionals behaves as $\sim s^{-2},{ }^{27}$ while the enhancement factor for the kinetic energy, as we will see later, must tend to $\sim s^{2}$.

\section{Functionals with correct asymptotic limits}

Since none of the above mentioned functionals, which correctly include the low gradient limit, Eq. (4), are able to reproduce the Kohn-Sham results in the surface regions of large gradients, we conclude that for these regions of space one must include a term that gives the correct kinetic energy in the limit of rapidly varying densities. It has been shown for many systems ${ }^{16}$ that in the limit $s \gg 1$ the noninteracting kinetic energy functional reduces to the von Weizsäcker functional ${ }^{29}$ given by

$$
t_{W}[n]=9 t^{(2)}[n] .
$$

This functional gives the exact kinetic energy for one- and two-electron systems. Furthermore, it becomes exact for weak and rapidly varying potentials, for exponentially decaying densities, and near the nuclear positions. Though there is no rigorous proof, it is believed that Eq. (9) represents the correct kinetic energy in the large $s[n]$ regime. $^{16}$ Therefore, an accurate noninteracting kinetic energy functional should reproduce both the slowly varying limit given by Eq. (4) and the rapidly varying limit given by Eq. (9).
Many attempts have been made to develop kinetic energy functionals that satisfy these two requirements. ${ }^{8} 9$ The Padé approximation used by DePristo and $\mathrm{Kress}^{8}$ leads to a functional that may be written

$$
t_{\mathrm{DK}}[n]=t^{(0)}(n) P_{4,3}(x),
$$

where $x$ is given by Eq. (8) and involves four semiempirical parameters determined by fitting the kinetic energy functional globally to the exact atomic total kinetic energies. At low $x$ values, the $P_{4,3}(x)$ enhancement function shown in Fig. 3 is slightly larger than the second order gradient expansion, at $x \approx 0.4$ it becomes smaller than $F_{\mathrm{GE}}^{2}(x)$, and for large $x$ values it increases towards an asymptotic behavior of $9 x$, which is the form corresponding to the functional given in Eq. (9).

The results for the surface kinetic energy given by $T_{\mathrm{DK}}[n]$ are presented in the eighth column of Table I. A comparison of the results obtained by the locally truncated functional $T_{L}[n]$ and the Padé approximation by DePristo and Kress shows that the accuracy of these functionals are very similar both for atoms (see Table II from Ref. 8) and for surfaces. This indicates that for the total kinetic energies of atoms the high gradient limit, inluded only in the latter functional, does not play an important role. Therefore, a functional of the form (10), where the Padé parameters have been obtained by fitting to Kohn-Sham atomic kinetic energies, does not provide a proper description of regions of high gradients, found for instance at surfaces, as may immediately be realized upon examination of Table I.

\section{E. The effect of a von Weizsäcker term}

The kinetic energy functional $t_{\mathrm{DK}}[n]$ fulfills both asymptotic limits, Eq. (4) and Eq. (9), but due to the parametrization based on atomic kinetic energies it reaches the large gradient limit only at very high $x$ values. In order to test directly the effect of a von Weizsäcker term on the kinetic energy of metal surfaces, we introduce the functional

$$
t_{\mathrm{GE} / W}[n]= \begin{cases}t^{(0)}[n]+t^{(2)}[n] & \text { if } x \leqslant \eta, \\ t_{W}[n] & \text { if } x>\eta,\end{cases}
$$

where $\eta$ is a parameter of order unity. Although this functional is clearly discontinuous at $x=\eta$, it reproduces the two asymptotic forms given by Eq. (4) (up to second order) and by Eq. (9).

The results obtained by means of $T_{\mathrm{GE} / W}[n]$ for $\eta=1$ are shown in Table I and in Fig. 1. It is immediately seen that the local inclusion of the von Weizsäcker term, where the gradient expansion fails, rather than the Thomas-Fermi term as in the locally truncated functional $T_{L}[n]$, reduces the errors of the second order gradient expansion by nearly one order of magnitude, and that of the fourth order gradient expansion by a factor of 3-5. Hence, the von Weizsäcker term appears to contain the most important contribution to the surface kinetic energy in the large $r_{s}$ regime. We note that in Eq. (6) the von Weizsäcker functional (9) is included when $\lambda=1$, but due to the presence of the Thomas-Fermi term this involves some amount of double counting especially for large $r_{s}$ values. 


\section{F. A kinetic energy functional for surfaces}

As a consequence of the above analysis of the effect of the von Weizsäcker term we propose the following simple kinetic energy functional:

$$
t_{3,2}[n]=t^{(0)}(n) P_{3,2}(x),
$$

where $x$ is given by Eq. (8), and

$$
P_{3,2}(x)=\frac{1+0.95 x+9 a x^{3}}{1-0.05 x+a x^{2}} .
$$

This functional is based on a one-parameter Padé approximation and for $a=0.396$ it gives the best possible surface kinetic energy for a wide range of densities. It is similar to but simpler than the semiempirical functional designed for atomic calculations by DePristo and Kress. ${ }^{8}$ At low gradients, $P_{3,2}(x)$ contains the dominant-wave-vector approximation similar to the functional of DePristo and Kress and it reduces to the second order gradient expansion at very low $x$ values, while for $x>1$ its slope rapidly reaches that of the von Weizsäcker functional, i.e., $9 x$.

The enhancement factor $P_{3,2}(x)$ is plotted in Fig. 3 and the kinetic energies obtained by means of this semiempirical functional may be found in the last column of Table I and in Fig. 1. It is seen that the functional reproduces the exact Kohn-Sham surface kinetic energies within $1 \%$ for a wide range of densities and gradients. We point out that since the semiempirical kinetic energy functional (12) in the low gradient limit reduces to the second order gradient expansion, and since the combination of a large gradient and a finite density, which leads to errors in surface calculation, does not occur in an isolated atom, or at least has little effect on the accuracy, the proposed functional may be used in atomic calculations with an accuracy similar to the first-principles kinetic energy functionals.

\section{CONCLUSION}

We have tested the accuracy of a number of semilocal noninteracting kinetic energy functionals in jellium model calculations for metal surfaces. It is found that the traditional gradient expansion, which provides an excellent description of the kinetic energy density inside the metallic part of a surface, where the Friedel oscillations occur, fails near the surface on account of the large gradient. We note that this large gradient limit cannot be described properly by fitting the parameters of a semiempirical functional only to atomic kinetic energies. We conlude that the slope of a true semilocal kinetic energy functional must reach the slope of the von Weizsäcker term already at relatively low values of the scaled gradient, i.e., $s[n] \approx 2.5-2.8$, and propose a simple semiempirical functional that fulfills this condition.

\section{ACKNOWLEDGMENTS}

One of us (L.V.) thanks Dr. N.D. Lang for the selfconsistent jellium surface computer code. The Center for Atomic-scale Materials Physics is sponsored by the Danish National Research Foundation. Part of this work was supported by the research project OTKA 016740 and 23390 of the Hungarian Scientific Research Fund.
${ }^{1}$ P. Hohenberg and W. Kohn, Phys. Rev. 136, B864 (1964).

${ }^{2}$ W. Kohn and L. Sham, Phys. Rev. 140, A1133 (1965).

${ }^{3}$ Dinesh Nehete, Vaishali Shah, and D. G. Kanhere, Phys. Rev. B 53, 2126 (1996).

${ }^{4}$ Michael Foley and Paul A. Madden, Phys. Rev. B 53, 10589 (1996).

${ }^{5}$ E. Smargiassi and P. A. Madden, Phys. Rev. B 49, 5220 (1994).

${ }^{6}$ L. Vitos, J. Kollár, and H. L. Skriver, Phys. Rev. B 55, 13521 (1997); L. Vitos, J. Kollár, and H. L. Skriver, ibid. 55, 4947 (1997)

${ }^{7}$ K. W. Jacobsen, J. K. Norskov, and M. J. Puska, Phys. Rev. B 35, 7423 (1987).

${ }^{8}$ Andrew E. DePristo and Joel D. Kress, Phys. Rev. A 35, 438 (1987).

${ }^{9}$ M. L. Plumer and M. J. Stott, J. Phys. C 18, 4143 (1985).

${ }^{10}$ Weitao Yang, Phys. Rev. A 34, 4575 (1986).

${ }^{11}$ Chengteh Lee and Robert G. Parr, Phys. Rev. A 35, 2377 (1987).

${ }^{12}$ E. W. Pearson and R. G. Gordon, J. Chem. Phys. 82, 881 (1985).

${ }^{13}$ Lin-Wang Wang and Michael P. Teter, Phys. Rev. B 45, 13196 (1992).

${ }^{14}$ P. García-González, J. E. Alvarellos, and E. Chacón, Phys. Rev. B 53, 9509 (1996).
${ }^{15}$ P. García-González, J. E. Alvarellos, and E. Chacón, Phys. Rev. A 54, 1897 (1996).

${ }^{16}$ R. M. Dreizler and E. K. U. Gross, in Density Functional Theory (Springer-Verlag, Berlin, 1990), and references therein.

${ }^{17}$ N. D. Lang and W. Kohn, Phys. Rev. B 1, 4555 (1970).

${ }^{18}$ E. P. Wigner, Phys. Rev. 46, 1002 (1934).

${ }^{19}$ C. Q. Ma and V. Sahni, Phys. Rev. B 16, 4249 (1977).

${ }^{20}$ Robert G. Parr and Weitao Yang, Density Functional Theory of Atoms and Molecules (Oxford University Press, Oxford, 1989), and references therein.

${ }^{21}$ A. Chizmeshya and E. Zaremba, Phys. Rev. B 37, 2805 (1988).

${ }^{22}$ N. L. Allan and D. L. Cooper, J. Chem. Phys. 84, 5594 (1986).

${ }^{23}$ Hsing Lee, Chengteh Lee, and Robert G. Parr, Phys. Rev. A 44, 768 (1991).

${ }^{24}$ A. Lembarki and H. Chermette, Phys. Rev. A 50, 5328 (1994).

${ }^{25}$ Daniel J. Lacks and Roy G. Gordon, J. Chem. Phys. 100, 4446 (1994).

${ }^{26}$ Ajit J. Thakkar, Phys. Rev. A 46, 6920 (1993).

${ }^{27}$ J. D. Perdew, in Electronic Structure of Solids, edited by P. Ziesche and H. Eschrig (Academic Verlag, Berlin, 1991), p. 11.

${ }^{28}$ J. P. Perdew, Phys. Lett. A 165, 79 (1992).

${ }^{29}$ C. F. von Weizsäcker, Ann. Phys. (N.Y.) 96, 431 (1935). 